agents are needed. (Thibeault-Eybalin M-P, Lortie A, Carmant L. Neonatal seizures: Do they damage the brain? Pediatr Neurol March 2009;40:175-180). (Respond: Dr Carmant, Epilepsy Clinic, Hospitalier Universitaire Sainte-Justine, 3175 Cote-Sainte-Catherine, Montreal, Quebec H3T 1C5, Canada. E-mail: 1ionel.carmant@umontreal.ca).

COMMENT. Although the experimental evidence from animal studies suggests that neonatal seizures can damage the developing brain, clinical reports are less convincing. Nonetheless, given the potential for seizure-induced brain damage, early and more effective treatment of neonatal seizures, including electrographic seizures, is generally advocated.

A study at Boston Children's Hospital on etiology and outcome of neonatal seizures found that global cerebral hypoxic-ischemia is the most frequent cause of neonatal seizures and a strong predictor of poor long-term outcome. (Tekgul $\mathrm{H}$ et al. Pediatrics 2006;117:1270-1280; Ped Neur Briefs April 2006;20:29-30). An abnormal neurologic examination in the neonatal period was an unreliable predictor of outcome.

Perilesional brain oedema and seizure activity: cause or effect? In response to a publication concerning seizures with calcified neurocysticercosis (Nash TE et al. Lancet Neurol 2008;7:1099-1105), Das A et al. question the proposed causative correlation of epileptogenesis and perilesional edema as an inflammatory response to calcified granulomas (Lancet Neurol 2009;8:225-226). Das et al agree that calcified lesions are seizure-causing foci but attribute the perilesional edema to the effect of the seizure per se. Reversible periictal MRI abnormalities are known to develop immediately after a seizure and may resolve within a few days or weeks. Their precise pathogenesis is unknown.

Hippocampal swelling demonstrated by MRI within 48 hours of a prolonged febrile seizure is transitory and thought to be caused by vasogenic edema. The swelling resolves within 5 days and the shrinkage of the hippocampus that follows at 4-8 months is thought to represent a preexisting developmental hippocampal abnormality that predisposes to the prolonged FS (Scott RC et al. Epilepsia 2006;47:1493-1498). Alternatively, the hippocampal shrinkage is consistent with brain damage caused by the seizure with subsequent development of mesial temporal sclerosis and epilepsy. (See Ped Neur Briefs Oct 2006;20:77; and Nov 2003;17:83).

\title{
UTILITY OF AMPLITUDE-INTEGRATED EEG IN THE NICU
}

The problem of artifacts in using the amplitude-integrated electroencephalogram (AIE) to assess cortical function in premature infants in the NICU were studied at Weill Cornell Medical College, New York, NY. A pair of standard EEG electrodes were attached to scalp frontotemporal areas of 10 infants. Impedance was maintained at $<10$ kohms. Continuous AIE recordings were performed for at least 60 min repeatedly in the first month. Artifacts were identified as large amplitude difference between jagged wave peaks and troughs. When the AIE tracing spikes upward in amplitude, the accompanying raw EEG during these segments was classified as artifact. Of 1683 segments of 48 recordings analyzed, $31 \%$ were normal brain waves, $60 \%$ were artifacts, and $8 \%$ indeterminate. No clinical or electrographic seizures were noted. Artifact related to muscle activity and electrode placement detracts from the value of the AIE in assessing cortical function in 
premature infants. (Suk D, Krauss AN, Engel M, Perlman JM. Amplitude-integrated electroencephalography in the NICU: frequent artifacts in premature infants may limit its utility as a monitoring device. Pediatrics Feb 2009;123:e328-e332). (Respond: Jeffrey M Perlman MB ChB, Weill Cornell Medical College, Department of Pediatrics, 525 E $68^{\text {th }}$ St, Suite N-506, New York, NY 10065. E-mail: jmp2007@med.cornell.edu).

COMMENT. Amplitude-integrated EEG (aEEG) is a sensitive monitor of background cerebral activity in the early prediction of outcome after perinatal asphyxia in term infants. Abnormal aEEG is used in selection of patients for hypothermia. The above study introduces a note of caution in the use of the aEEG as an indicator of cortical function in premature infants in the NICU. The concurrent use of the conventional EEG and careful attention to electrode placement are suggested.

\section{EVALUATION OF SLEEP DEPRIVATION IN THE PEDIATRIC EEG}

The value of sleep deprivation to increase the yield of routine outpatient EEG in the diagnosis of epilepsy was assessed in a randomized, blinded comparison of routine EEG (REEG) versus sleep-deprived EEG (SDEEG) in 206 children aged 0 to 18 years referred by neurologists at Helen DeVos Children's Hospital, Grand Rapids, MI. Patients referred for EEG had $>1$ seizure $(83 \%)$ or unclear spells $(17 \%)$. Before the EEG, sleep-deprived patients averaged 4.9 hours sleep while those not sleep deprived had 7.9 hours sleep $(\mathrm{P}<.00001)$. Only $48 \%$ of the REEG group had adequate sleep for age vs $12 \%$ of the SD group $(\mathrm{P}<.0001)$. Of a total of 127 (64\%) patients who fell asleep during the EEG, 73\% of SDEEG group reached stage 2 sleep vs $55 \%$ of REEG group $(\mathrm{P}=.009)$. EEGs were normal in $67.7 \%$ of the REEG group vs $55.6 \%$ of SDEEG group $(\mathrm{P}=.08)$. Epileptiform discharges were recorded in $35 \%$ of REEG group vs $40 \%$ of SDEEG patients. No specific type of EEG abnormality was significantly more prevalent in the SDEEG group. Abnormal EEGs were more frequent in patients with clinically diagnosed seizures compared with those with unclear spells $(\mathrm{P}=.001)$. Odds of an abnormal EEG were also higher in the older ( $>3$ years) age group vs younger children $(\mathrm{P}=.022)$. Sleep during the EEG did not influence the proportion of patients with epileptiform records in comparison with awake EEG only. Sleep deprivation, but not sleep during the EEG, provides a modest increase in EEG epileptiform discharges in children diagnosed with seizures by neurologists. Compared with a routine EEG, 11 SDEEGs would be required to identify 1 additional child with epileptiform discharges on EEG, (DeRoos ST, Chillag KL, Keeler M, Gilbert DL. Effects of sleep deprivation on the pediatric electroencephalogram. Pediatrics Feb 2009;123:703-708). (Respond: Steven T DeRoos MD, 1300 Michigan, Suite 102, Grand Rapids, MI 49503. E-mail: steven.deroos@devoschildrens.org).

COMMENT. Routine EEG fails to confirm a diagnosis of epilepsy in approximately $50 \%$ of patients with clinical seizures. Sleep and sleep deprivation are recommended to enhance the diagnostic yield. Despite the burden on parents and child of following the protocol of sleep deprivation (Nijhof SL et al, 2005), many authorities consider the benefits of sleep-deprived EEG (SD-EEG) to outweigh the inconvenience. Some studies that support the use of SD include Rowan AJ et al (1982), Klingler D (1982), and Leach JP et al (2006) who compared the yield of EEG abnormalities in 85 patients using 3 different protocols. 